\title{
Efficient light management scheme for thin film silicon solar cells via transparent random nanostructures fabricated by nanoimprinting
}

\author{
Corsin Battaglia, ${ }^{\text {a) }}$ Karin Söderström, Jordi Escarré, Franz-Josef Haug, Didier Dominé, \\ Peter Cuony, Mathieu Boccard, Grégory Bugnon, Céline Denizot, Matthieu Despeisse, \\ Andrea Feltrin, and Christophe Ballif \\ Institute of Microengineering (IMT), Photovoltaics and Thin Film Electronics Laboratory, \\ Ecole Polytechnique Fédérale de Lausanne (EPFL), 2000 Neuchâtel, Switzerland
}

(Received 15 February 2010; accepted 4 May 2010; published online 24 May 2010)

\begin{abstract}
We propose the use of transparent replicated random nanostructures fabricated via nanoimprinting on glass as next-generation superstrates for thin film silicon solar cells. We validate our approach by demonstrating short-circuit current densities for $p-i-n$ hydrogenated microcrystalline silicon solar cells as high as for state-of-the-art nanotextured $\mathrm{ZnO}$ front electrodes. Our methodology opens exciting possibilities to integrate a large variety of nanostructures into $p-i-n$ solar cells and allows to systematically investigate the influence of interface morphology on the optical and electronic properties of the device in order to further improve device performance. (ㅇ 2010 American Institute of Physics. [doi:10.1063/1.3432739]
\end{abstract}

Intensive research over the past 15 years has identified the so-called micromorph tandem solar cell, a stack of a high-gap amorphous (a-Si:H) and a low-gap microcrystalline $(\mu \mathrm{c}-\mathrm{Si}: \mathrm{H})$ silicon solar cell on a glass superstrate, as one of the most promising thin film technologies for lowering the cost of photovoltaic electricity, ${ }^{1}$ as it is based on abundant, nontoxic materials and low-temperature processes. Advanced light management concepts are crucial to further improve conversion efficiencies of thin film silicon solar cells, as the absorption coefficient of silicon is small in the near-infrared region. The most common approach to improve optical performance is by means of light scattering at randomly textured interfaces. However, neither the ideal interface morphology, respecting restrictions imposed by the growth of the silicon layers, ${ }^{2}$ nor the ideal scattering characteristics, which maximizes the photoinduced current in the cell, have been identified to date.

For micromorph cells in the $p-i-n$ configuration, light trapping is traditionally achieved by scattering at the interface between the transparent front electrode and the absorbing silicon layers by exploiting either the natural, randomly oriented pyramidal texture of $\mathrm{ZnO}$ grown via low-pressure chemical vapor deposition (LP-CVD) (Ref. 3) or the craterlike texture of sputtered $\mathrm{ZnO}$ obtained after wet-etching in $\mathrm{HCl}^{4}{ }^{4}$ These approaches have two following major drawbacks: (1) although the characteristic size of the features can be adjusted by adapting growth or etch conditions, the basic feature morphology is given by the underlying physical process. (2) As the $\mathrm{ZnO}$ layer simultaneously serves as light scatterer and electrical front contact, optical and electrical properties are interlinked and cannot be studied and optimized completely independently.

In this paper, we propose to transfer the task of light scattering for $p-i-n$ solar cells to transparent replicated textures on glass fabricated via ultraviolet (UV) nanoimprinting. Our approach has the advantage of separating the optical from the electrical task of the front contact and allows a large range of morphologies to be implemented as front scatterers.

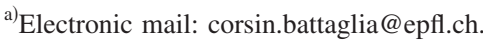

Most interestingly, this opens the door to exciting possibilities as not only transparent structures may serve as master for the replication, but also structures from opaque materials, such as hot silver, ${ }^{5}$ a classical structure so far exclusively used for $n$-i- $p$-type solar cells. Also more exotic nanostructures such as self-ordered dimple patterns ${ }^{6}$ or nanodomes, ${ }^{7}$ which have shown impressive results in the $n-i-p$ configuration, may be transferred from $n-i-p$ to $p-i-n$. Furthermore, criteria, derived theoretically within scalar scattering theory ${ }^{8}$ and rigorous diffraction theory, ${ }^{9}$ on how a random interface should look like to maximize light absorption and device performance, may be tested experimentally.

Here we validate our approach by demonstrating shortcircuit currents for cells grown on the replicated structures comparable to those grown directly on the master taking the pyramidal structure of LP-CVD ZnO, known for its excellent light trapping properties, ${ }^{10}$ as an example. Whereas the blue and green part of the solar spectrum is well absorbed by the amorphous top-cell of the micromorph tandem junction, the microcrystalline bottom-cell, absorbing the red and nearinfrared part of the spectrum up to $1100 \mathrm{~nm}$, relies heavily on efficient light trapping. For this reason we choose to study single junction $\mu \mathrm{c}-\mathrm{Si}: \mathrm{H}$ cells, whose external quantum efficiency (EQE) may be measured more directly.

Pyramidally textured boron-doped $\mathrm{ZnO}$ layers with resistivity $\rho=4 \times 10^{-3} \Omega \mathrm{cm}$, carrier density $n=4$ $\times 10^{19} \mathrm{~cm}^{-3}$ and thickness $t=4.8 \mu \mathrm{m}$ serving as a reference substrate for the cells and as a master for the replication process were grown via LP-CVD (Ref. 3) on $0.5 \mathrm{~mm}$ thick borosilicate glass without antireflection coating and subjected to 20 min plasma treatment. ${ }^{10}$ The basic UV nanoimprinting replication process will be described in Ref. 11. A significantly improved version of this process allows us to obtain replicas of very high fidelity and will be described elsewhere. ${ }^{12}$ Sputtered indium tin oxide (ITO) $\left(\rho=3 \times 10^{-4} \Omega \mathrm{cm}, n=6 \times 10^{20} \mathrm{~cm}^{-3}, \mathrm{R}_{\text {sheet }}=42 \Omega_{\square}\right.$, and $t=70 \mathrm{~nm})$, covered by a thin sputtered aluminum-doped $\mathrm{ZnO}$ layer $(t=20 \mathrm{~nm})$ served as a front contact for the replica. All $p-i-n \mu \mathrm{c}-\mathrm{Si}: \mathrm{H}$ cells, of size $5 \times 5 \mathrm{~mm}^{2}$ with intrinsic layer thickness of $2.0 \mu \mathrm{m}$, were deposited simulta- 

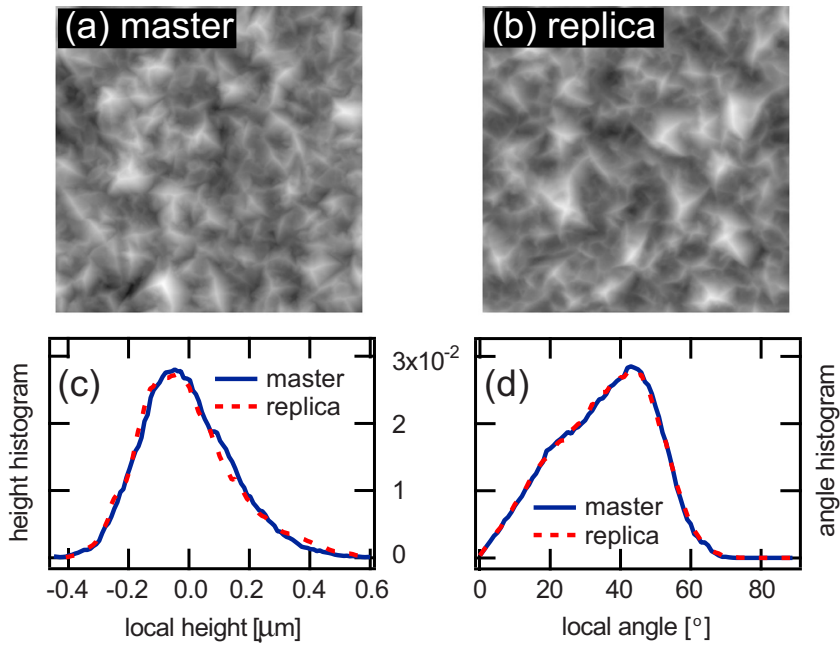

FIG. 1. (Color online) AFM topographies of $\mathrm{ZnO}$ master (a) and its transparent replica (b), image size $10 \times 10 \mu \mathrm{m}^{2}$ with the extracted local height (c), and local angle (d) histograms.

neously by plasma-enhanced chemical vapor deposition. Short-circuit current density $\left(J_{\mathrm{sc}}\right)$, open-circuit voltage $\left(V_{\mathrm{oc}}\right)$, and fill factor (FF) of the $\mu \mathrm{c}-\mathrm{Si}: \mathrm{H}$ cells were determined as in Ref. 10 under standard test conditions.

Surface morphologies were measured using atomic force microscopy (AFM) probing an image size of $10 \times 10 \mu \mathrm{m}^{2}$ with a resolution of $512 \times 512$ pixels. Angle-resolved scattering (ARS) of light was measured with a detector on a home-built goniometer under normal incidence onto the glass side, using a laser at a wavelength of $543 \mathrm{~nm}$. The spectral dependence of total and diffuse optical transmittance was measured with a photospectrometer equipped with an integrating sphere. Simulated optical curves were calculated using the methodology recently presented in Ref. 13. Our model makes use of a slightly modified RayleighSommerfeld diffraction integral proposed by Harvey ${ }^{14}$ and requires only measured AFM surface profile data and the refractive index as input.

Figure 1 presents AFM images of the master and replicated $\mathrm{ZnO}$ surfaces with their pyramidal structure. In order to quantify the quality of the replication, we compare the local height and angle histogram extracted from these two images in Figs. 1(c) and 1(d), respectively. Both histograms are practically identical for master and replica indicating the high fidelity of the replication process.

In Figs. 2(a) and 2(b) we show the experimental ARS curves for the master and replicated structure, respectively. It is important to note that the thin conformal ITO layer on top of the replicated structure does only slightly alter the angular and spectral scattering behavior (not shown). The angular dependence for scattering into air behaves quite different for the two structures, with the replica scattering more light into smaller angles than the master. In order to understand the origin for this difference we also present calculated ARS curves. Both curves were obtained taking the AFM image of the master in Fig. 1(a) as input, but using different refractive indices in the calculation, to take into account the optical properties of the scatterer (refractive index $n=2$ for $\mathrm{ZnO}$ and $n=1.5$ for the replica). The calculated curves nicely reproduce the two different behaviors of the ARS curve, which can therefore be attributed to the difference in refractive index. We note however that they deviate slightly at large scat-
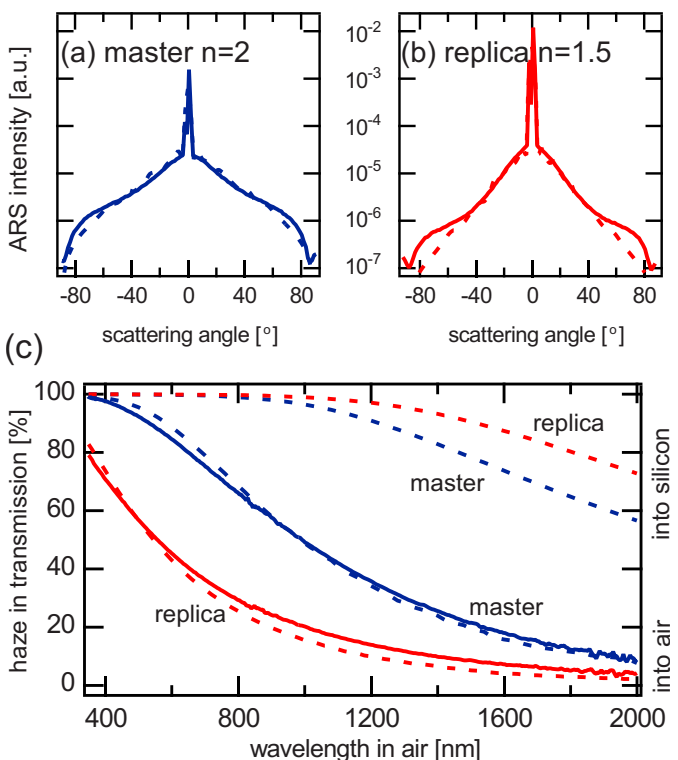

FIG. 2. (Color online) Experimental and calculated ARS curves for the master (a) and its replica (b) for scattering into air. (c) Experimental and calculated haze curves for the master and its replica for scattering into air and into silicon. Experimental curves are shown as continuous lines, calculated curves as dashed lines. For the calculated curves for the replica, the AFM image of the master in Fig. 1(a) was used, but with the refractive index $n=1.5$ of the replica.

tering angles, presumably because we model the optical interface by a random phase-screen with zero thickness which neglects diffraction within the peak to valley depth of the rough surface. ${ }^{13}$

In Fig. 2(c) we focus on the spectral dependence of the scattered light. Experimentally, the haze in transmission is determined by taking the ratio between diffuse and total transmittance. Theoretically, we can derive the haze value at a given wavelength by integrating the calculated ARS intensity without the specular peak over the full hemisphere and normalizing it by the total intensity. Inspection of Fig. 2(c) shows that the replica scatters significantly less light into air $(n=1)$ than the master due to its lower refractive index. However, although not accessible experimentally, the calculated haze curves for transmission into silicon $(n=4)$, show that the replica $(n=1.5)$ scatters slightly more light than the master $(n=2)$ due to the increased contrast in refractive index.

We finally turn to the experimental results for the $\mu \mathrm{c}-\mathrm{Si}: \mathrm{H}$ cells grown on the master and the replica. Figure 3 compares the EQE curves for the two cells. The associated $J_{\text {sc }}$ 's extracted from the EQE's are given in Table I, with the

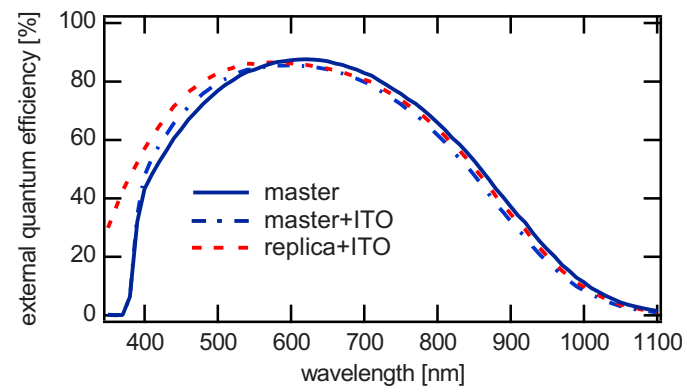

FIG. 3. (Color online) EQE of $p-i-n \mu \mathrm{c}-\mathrm{Si}: \mathrm{H}$ solar cells fabricated on master and replica. 
TABLE I. Characteristics of $p-i-n \mu \mathrm{c}-\mathrm{Si}: \mathrm{H}$ solar cells fabricated on master and replicated substrate.

\begin{tabular}{lccccc}
\hline \hline Substrate & $\begin{array}{c}J_{\text {sc }}^{350-1100 \mathrm{~nm}} \\
\left(\mathrm{~mA} / \mathrm{cm}^{2}\right)\end{array}$ & $\begin{array}{c}J_{\mathrm{sc}}^{650-1100 \mathrm{~nm}} \\
\left(\mathrm{~mA} / \mathrm{cm}^{2}\right)\end{array}$ & $\begin{array}{c}V_{\mathrm{oc}} \\
(\mathrm{mV})\end{array}$ & $\begin{array}{c}\mathrm{FF} \\
(\%)\end{array}$ & $\begin{array}{c}\text { Efficiency } \\
(\%)\end{array}$ \\
\hline Master & 25.1 & 12.9 & 507 & 71 & 9.0 \\
Master+ITO & 24.3 & 12.0 & 488 & 69 & 8.2 \\
Replica+ITO & 25.4 & 12.5 & 504 & 57 & 7.3 \\
\hline \hline
\end{tabular}

replica producing the highest current. In the red and infrared part, where light trapping is most relevant, the EQE of the replica is only slightly lower than for its master. This difference, accounting for $0.4 \mathrm{~mA} / \mathrm{cm}^{2}$ between 650 and $1100 \mathrm{~nm}$ (see Table I), can be attributed to the much stronger free carrier absorption (FCA) of ITO in this spectral range compared to $\mathrm{ZnO}$. Recently Koida et al. ${ }^{15}$ developed a promising hydrogen-doped indium oxide $\left(\operatorname{In}_{2} \mathrm{O}_{3}: \mathrm{H}\right)$ with very high near-infrared transparency, which allows to circumvent most of the current losses associated with the FCA of ITO. To get a quantitative estimate of how much more current we could gain using $\operatorname{In}_{2} \mathrm{O}_{3}: \mathrm{H}$ instead of ITO, we deposited a cell on the $\mathrm{ZnO}$ master with an additional ITO layer. Its EQE falls below the EQE of the replica, one more evidence for the high quality of the replica. Inspection of Table I reveals that above $650 \mathrm{~nm}$ up to $0.9 \mathrm{~mA} / \mathrm{cm}^{2}$ of current can be gained by avoiding FCA.

Below $400 \mathrm{~nm}$, the EQE for the master drops to $0 \%$ as the band gap of $\mathrm{ZnO}$ is around $380 \mathrm{~nm}$, whereas ITO exhibits a larger band gap at $330 \mathrm{~nm}$. In the blue and green part of the EQE, the replica clearly performs better than its master. Interestingly we see that also the master with the ITO layer experiences a slight increase in EQE with respect to the master without ITO in this spectral range. We attribute this effect to the modified nucleation of the silicon layer at the different front contacts. ${ }^{16}$

Table I summarizes the electrical characteristics of our solar cells. The FF of the replica is relatively low, which severely affects its apparent efficiency. This is due to significant series resistance losses in the ITO caused by the high current densities produced in the $\mu \mathrm{c}-\mathrm{Si}: \mathrm{H}$ cell. However, once integrated as a bottom cell into a micromorph tandem device those Ohmic losses become negligible as the microcrystalline cell produces half of the current because of current matching between the two component cells.

An important feature of our approach is its compatibility with industrial in-line mass production. The lacquer used for the replication can in principle easily be applied on large glass panels. The glass panels are then passed below a stamp roll transferring the nanostructure onto the lacquer, which is subsequently cured under UV light. We were not able to observe any significant degradation of the optical properties of our lacquer under prolonged UV light and humidity exposure, which is important for the long-term stability of the solar module. Thanks to the negligible absorbance of the lacquer, which behaves optically identically to glass, the use of transparent replicated nanostructures is furthermore compatible with laser scribing, allowing for a monolithic series interconnection of cell segments to large area modules.

In summary, we presented the high potential of transparent random nanostructures fabricated via nanoimprinting for the use in thin film silicon solar cells. Replicating the pyramidal structure of LP-CVD $\mathrm{ZnO}$, we are able to obtain shortcircuit current densities for $\mu \mathrm{c}-\mathrm{Si}: \mathrm{H}$ solar cells as high as for the master structure. A further increase in current is expected when replacing ITO by $\operatorname{In}_{2} \mathrm{O}_{3}: \mathrm{H}$. Our approach furthermore allows the incorporation of so far unexplored morphologies into the cells and to systematically study the influence of interface morphology on the optical and electronic characteristics of the cells.

The authors acknowledge the support of the Swiss Federal Energy Office under Project No. 101191.

${ }^{1}$ A. G. Aberle, Thin Solid Films 517, 4706 (2009).

${ }^{2}$ M. Python, E. Vallat-Sauvain, J. Bailat, D. Dominé, L. Fesquet, A. Shah, and C. Ballif, J. Non-Cryst. Solids 354, 2258 (2008).

${ }^{3}$ S. Faÿ, J. Steinhauser, N. Oliveira, E. Vallat-Sauvain, and C. Ballif, Thin Solid Films 515, 8558 (2007).

${ }^{4}$ M. Berginski, J. Hüpkes, W. Reetz, B. Rech, and M. Wuttig, Thin Solid Films 516, 5836 (2008).

${ }^{5}$ A. Banerjee and S. Guha, J. Appl. Phys. 69, 1030 (1991).

${ }^{6}$ H. Sai, H. Fujiwara, M. Kondo, and Y. Kanamori, Appl. Phys. Lett. 93, 143501 (2008).

${ }^{7}$ J. Zhu, C.-M. Hsu, Z. Yu, S. Fan, and Y. Cui, "Nanodome Solar Cells with Efficient Light Management and Self-Cleaning," Nano Lett. (to be published).

${ }^{8}$ J. Krč, M. Zeman, F. Smole, and M. Topič, J. Appl. Phys. 92, 749 (2002).

${ }^{9}$ C. Rockstuhl, S. Fahr, F. Lederer, K. Bittkau, T. Beckers, and R. Carius, Appl. Phys. Lett. 93, 061105 (2008).

${ }^{10}$ J. Bailat, D. Dominé, R. Schlüchter, J. Steinhauser, S. Faÿ, F. Freitas, C. Bucher, L. Feitknecht, X. Niquille, T. Tscharner, A. Shah, and C. Ballif, Proceedings of the Fourth World Conference on Photovoltaic Energy Conversion, 2006, p. 1533.

${ }^{11}$ K. Söderström, J. Escarré, O. Cubero, F.-J. Haug, S. Perregaux, and C. Ballif, "UV-nano-imprint lithography for the replication of back reflectors for $n-i-p$ thin film silicon solar cells," Prog. Photovolt: Res. Appl. (to be published).

${ }^{12}$ J. Escarré, K. Söderström, F.-J. Haug, C. Battaglia, and C. Ballif, "High fidelity transfer of nanometric textures by UV embossing for thin film solar cell applications" (unpublished).

${ }^{13}$ D. Dominé, F.-J. Haug, C. Battaglia, and C. Ballif, J. Appl. Phys. 107, 044504 (2010).

${ }^{14}$ J. E. Harvey, C. L. Vernold, A. Krywonos, and P. L. Thompson, Appl. Opt. 38, 6469 (1999)

${ }^{15}$ T. Koida, H. Fujiwara, and M. Kondo, Jpn. J. Appl. Phys., Part 2. 46, L685 (2007)

${ }^{16}$ E. Vallat-Sauvain, J. Bailat, J. Meier, X. Niquille, U. Kroll, and A. Shah, Thin Solid Films 485, 77 (2005). 